\title{
Adaptive Load Shedding via Fuzzy Control in Data Stream Management Systems
}

\author{
Can Basaran*, Kyoung-Don Kang ${ }^{\dagger}$, Yan Zhou ${ }^{\ddagger}$ and Mehmet H. Suzer ${ }^{\S}$ \\ *Dept. of Inf. and Comm. Eng., Daegu Gyeongbuk Institute of Science \& Technology (DGIST), Korea \\ Email: cbasaran@dgist.ac.kr \\ ${ }^{\dagger}$ Dept. of Comp. Sci., Binghamton University, Email: kang@binghamton.edu \\ ${ }^{\ddagger}$ Cisco Systems, Email: yazhou2@cisco.com

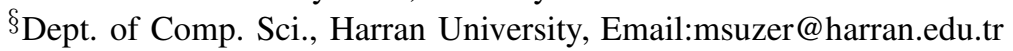

\begin{abstract}
Data stream management systems (DSMS) aim to process massive data streams in a timely fashion to support important applications, e.g., financial market analysis. However, DSMS can be overloaded due to large bursts in data stream arrivals and data-dependent query executions. To avoid overloads, we design a new load shedding scheme by applying distributed fuzzy logic control, which is very effective to deal with uncertainties in highly dynamic systems such as DSMS, based on the per-stream backlog and selectivity of each query operator. We have implemented our approach by extending an open source distributed DSMS. The performance evaluation using high-rate Internet traces shows that our approach closely supports a specified backlog bound for each data stream queue, while improving the query processing delay, with little overhead.
\end{abstract}

\section{INTRODUCTION}

Data stream management systems (DSMS), including [1], [2], [3], [4], [5], [6], are developed to efficiently process data streams in important applications, e.g., network monitoring, financial market analysis, and transportation management. As data streams may be unbounded and arrive at high rates, most DSMS support continuous reevaluation of queries over sliding (or jumping) windows of stream data tuples, e.g., stock price or network monitoring data streams. Using a declarative language (e.g., a windowed SQL-like language [5] or graphical query processing boxes [4]) provided by a DSMS, a user can easily write a DSMS application, which is interpreted as a graph of the interconnected continuous query (CQ) operators necessary to process data streams for the application.

Ideally, DSMS are desired to process data streams with a minimal delay to monitor critical real world phenomena, e.g., market status changes or denial of service (DoS) attacks. Ironically, supporting this property is most challenging when it is most needed. For example, a large amount of data streams may arrive in a short time interval when the status of a financial market or communication network is widely fluctuating. Thus, the DSMS can be overloaded, resulting in late detection of DoS attacks or losses of business opportunities. To deal with potentially bursty arrivals of stream data, a CQ operator, e.g., a windowed select, project, join, or aggregation operator, usually buffer its input data streams in memory. Under overload, however, the input stream queues for buffering may grow rapidly. As a result, the system may suffer from excessive data losses or CQ processing delays. It may even become unreliable due to potential thrashing caused by severe memory contention.

To address the problem, we design a novel load shedding scheme to systematically control the backlog accumulated by each stream even in the presence of dynamic workloads. For load shedding, a DSMS should determine 1) when to shed load, 2) where to shed load, 3) which stream data tuples to drop, and 4) how much load to shed [7]. Concerning 1) and 2), we take an immediate, localized approach to load shedding. Our load shedding scheme runs immediately when the amount of backlog of any individual data stream changes by more than a specified threshold. It probabilistically drops tuples, if necessary, to control per-stream backlog to be below the desired bound without requiring any global coordination, which is computationally expensive and time consuming in distributed DSMS. By doing this, our approach avoids a potential avalanche, in which the backlog accumulated in a stream queue may largely delay the succeeding CQ operators in a cascading manner. Further, the impact of tuple dropping is evenly spread across the query operators cooperating for a specific application. If load shedding is applied only to the data stream sources, too many queries can be affected, since one stream may fan out to multiple streams [7]. On the other hand, only performing load shedding at the sinks of the query graph, which produce the final CQ processing results, is subject to excessive resource waste caused by producing a large amount of intermediate data streams that will be eventually dropped.

Regarding 3) and 4), we drop tuples randomly according to the drop probability, because random tuple dropping incurs a minimal decrease of the accuracy of query processing results without making any statistical assumptions about data distributions [5], [7]. ${ }^{1}$ To determine how much load to shed, we apply fuzzy control techniques [9] to derive the drop probability considering the current DSMS status. Fuzzy control is based on fuzzy logic developed to deal with uncertainties prevalent in real world applications. Due to its robustness, it is widely applied to support performance and safety features in many important systems including automobiles and consumer electronics. Since it can naturally express fuzzy (uncertain)

\footnotetext{
${ }^{1}$ Our approach is not limited to random dropping. If the utilities of the data streams are known a priori similar to [8], our approach can be easily adapted to drop the tuple with the smallest utility first.
} 
concepts that are not completely true or false but partially true, it is very effective to handle uncertainties in highly-dynamic, nonlinear systems. In this paper, we apply it to efficiently handle uncertainties in DSMS including bursty data arrivals and aperiodic, data-dependent CQ executions.

Fuzzy control is performed using if-precedent-thenconsequent rules derived based on the logical understanding of the fundamental characteristics of the controlled system, e.g., a DSMS. We design a set of novel fuzzy rules to dynamically adjust the per-stream drop probability, if necessary, to control the length of each stream queue to be below the specified set-point based on the queue length error, i.e., the difference between the queue length set-point and current length of any individual data stream queue in a DSMS, even in the presence of dynamic workloads. Moreover, we design another set of new fuzzy rules to consider not only the queue length error but also the dynamics of query operators to drop just enough tuples, if necessary, to avoid overloads. Specifically, we consider the selectivity of each individual query operator, i.e., the ratio of the number of the output stream tuples produced by an operator to the number of the input stream tuples entering the operator, to further adjust the shedding probability. We seamlessly integrate the per-stream queue length and selectivity controllers into a single load shedding framework to avoid both overloads and excessive data losses in DSMS. For example, even when a data stream queue is longer than the desired bound, our approach does not increase the shedding probability for the specific stream, if a large fraction of the stream is filtered out by a preceding query operator before the stream reaches the queue being controlled (or vice versa). ${ }^{2}$

In addition, our fuzzy logic scheme for load shedding is executed in an event-driven manner; that is, it is executed when the length of any queue, i.e., per-stream backlog, changes by more than the specified event threshold. Being event-driven, our load shedding scheme automatically executes more often when the per-stream backlog increases or vice versa unlike the other control theoretic techniques using a fixed control period [10], [11], [12]. Further, our approach requires neither centralized control nor global information about the system status. For each stream queue, the shedding probability is adjusted considering the per-stream queue size error and selectivity of the preceding CQ operator. In summary, our approach supports immediate, localized per-stream backlog control in an event-driven manner to control the size of each data stream buffer to be below a specified set-point even in the presence of uncertainties. Supporting these features is not directly considered by previous work on DSMS including [5], [4], [6], [13], [14], [15], [8], [16].

For performance evaluation, we have implemented our approach by extending one of the most advanced open source DSMS, Borealis [4], in a Linux cluster. For performance

\footnotetext{
${ }^{2}$ Note that network congestion control is not directly applicable to load shedding in DSMS [7], since it simply drops packets as the buffer in a network router builds up without simultaneously considering the aforementioned four issues and CQ selectivity important in DSMS.
}

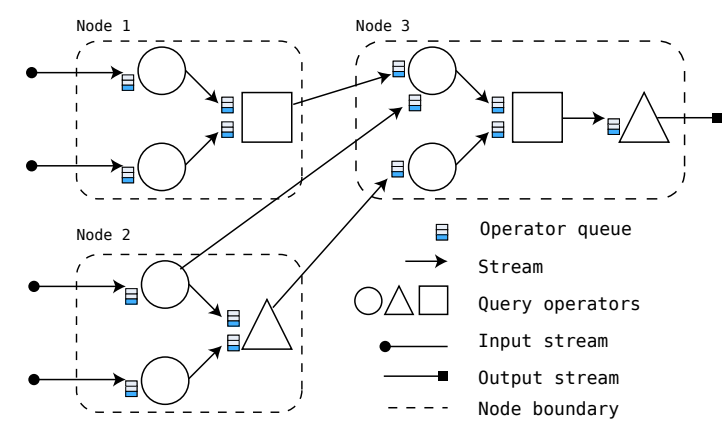

Fig. 1. A Continuous Query Tree

comparisons between our approach and Borealis, we use highspeed Internet traces [17] different from most existing research on DSMS (including [5], [4], [6], [18], [19], [13], [14], [15], [8], [16]), which uses synthetic workloads or outdated traces such as [20], [21]. The performance evaluation results show that our approach closely supports the desired per-stream queue length set-point. The per-stream queue length of our approach is up to two orders of magnitude shorter than the one supported by Borealis [16]. In addition, our approach considerably decreases the delay for query processing.

Notably, our approach is lightweight. It consumes less than $1 \% \mathrm{CPU}$ utilization and $1 \mathrm{~KB}$ of memory to store the if-then rules for systematic load shedding. It has little overhead, since it is localized and requires no global feedback or adaptation across the entire query graph (also called a query network in the DSMS literature). Further, it only requires simple computations and look-ups of the fuzzy rules stored in two tables to compute the tuple drop probability for load shedding.

The rest of the paper is organized as follows. An overview of the DSMS architecture and load shedding in DSMS is given in Section II. A description of our load shedding scheme and fuzzy rule design is given in Section III. Performance of our approach is evaluated in Section IV. Section V discusses related research. Finally, Section VI concludes the paper and discusses future work.

\section{SyStem ARCHITECTURE}

In a distributed DSMS application, the stream sources provide input data streams to the rest of the nodes and the final output is produced at one or more sink nodes as shown in Figure 1. CQ operators, e.g., windowed select, project, or join, process data streams and produce intermediate data streams. A query is a tree of CQ operators. One or more queries can be linked together to form a query graph/network as shown in Figure 1. In DSMS, a queue is usually assigned to buffer data arriving from an individual data stream [4], [22], [5]. The stream queues in one node of a distributed DSMS usually share a single memory pool in the node without capping the length of an individual queue. Thus, a sudden arrival rate increase in a single stream can cause the other operators to starve due to the insufficient memory. As a result, the overall response time may increase due to the data and control dependencies in the query network. 
To prevent a subset of streams from consuming excessive amounts of memory, we directly control the backlog accumulated in each stream queue to be below a specified threshold, $Q_{S}$, even in the presence of bursty stream data arrivals and aperiodic, data-dependent executions of query operators. ${ }^{3}$ Since the arrival rate and selectivity may vary from stream to stream and operator to operator, our approach to load shedding performs on a per-stream, per-CQ operator basis.

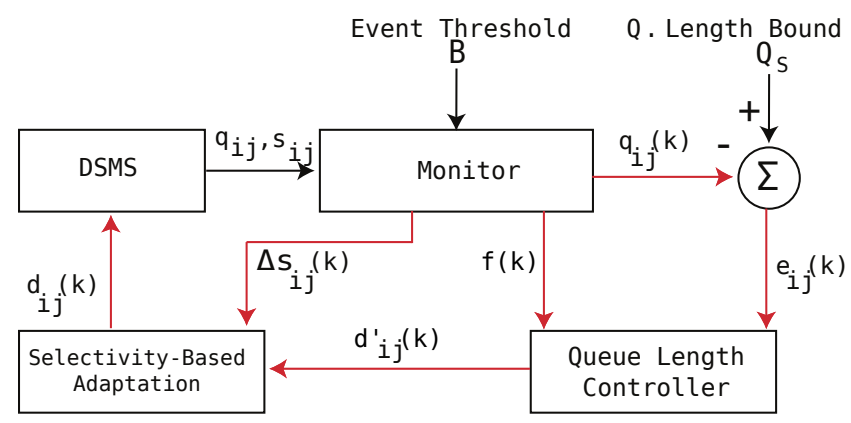

Fig. 2. Adaptive Load Shedding

Figure 2 shows the high level structure of our adaptive load shedding scheme. Let $I_{i j}$ represent stream $j$ flowing into CQ operator $i$ in a distributed DSMS. In Figure 2, $q_{i j}$ represents the instantaneous length of the queue used to buffer $I_{i j}$ 's tuples and $q_{i j}(k)$ represent the size of the queue at the $k^{t h}$ control event that occurs when:

$$
\left|q_{i j}(k)-q_{i j}(k-1)\right| \geq B
$$

where $k \geq 1$ and $B$ is the control event threshold used to detect a noteworthy increase or decrease of $q_{i j}$. (We assume that $q_{i j}(0)=0 \forall i, j$ when the system starts initially.) We take an event-driven approach, since it is infeasible to find one common control period effective to monitor and adapt all streams, which may have significantly different average and transient arrival rates.

To control the length of each stream queue to be below the specified set-point $Q_{s}$, we employ two interconnected controllers: a queue length control module (QCM) and a selectivity-based control module (SCM) in Figure 2. Via fuzzy control, QCM computes the drop probability $d_{i j}^{\prime}(k)$. SCM computes $d_{i j}(k)$ actually used to probabilistically drop tuples of $I_{i j}$, if necessary, to support $Q_{s}$. To compute $d_{i j}(k)$, SCM adjusts $d_{i j}^{\prime}(k)$ considering the selectivity of the preceding CQ operator, which pushes stream data tuples into $I_{i j}$ 's queue.

Whenever a control event occurs for an arbitrary stream in a DSMS, we apply our load shedding method to the specific stream. Therefore, in the rest of this paper, we consider only a single stream $I_{i j}$ to present our per-stream load shedding scheme. We omit the operator and stream indexes $i$ and $j$ for brevity without loss of generality.

\footnotetext{
${ }^{3}$ For the clarity of presentation, we use a system-wide queue length bound $Q_{S}$ that applies to every data stream queue in a DSMS. However, our approach is not limited to a single queue length bound. Different streams may have different queue length bounds.
}

At the $k^{t h}$ (control) event, i.e., when $|q(k)-q(k-1)| \geq B$, QCM computes the error:

$$
e(k)=\left(Q_{s}-q(k)\right) / Q_{s} .
$$

At the $k^{\text {th }}$ event, QCM in Figure 2 measures the time between two consecutive events:

$$
\Delta t(k)=t(k)-t(k-1)
$$

where $t(k)$ indicates the time at which the $k^{t h}$ event occurs.

QCM also computes the queue length change between the consecutive events:

$$
\Delta q(k)=q(k)-q(k-1) .
$$

Using Eq 3 and Eq 4, QCM calculates the trend of the queue length change representing the speed and direction of the queue length change:

$$
f(k)=\left\{\begin{aligned}
\Delta t(k) & \text { if } \Delta q(k)>0 \\
-\Delta t(k) & \text { if } \Delta q(k)<0
\end{aligned}\right.
$$

Note that $\Delta q(k) \neq 0$ when $f(k)$ is computed, since a control event occurs only when the queue length changes by more than $B$ as defined in $\mathrm{Eq} \mathrm{1}$. By considering the trend in addition to the error, we intend to improve the effectiveness of per-stream backlog management in DSMS. For example, even when the queue length is longer than $Q_{s}$, it is unnecessary to increase the tuple drop probability by a large amount, if the queue length is decreasing fast or vice versa. Logical observations such as these ones can be easily formulated via fuzzy rules. Based on $e(k)$ and $f(k)$, QCM computes the drop probability $d^{\prime}(k)$ using the fuzzy rules (described in Section III).

As shown in Figure 2, the output of QCM, $d^{\prime}(k)$, is an input to SCM that adapts $d^{\prime}(k)$, if necessary, to further enhance the effectiveness of load shedding by considering the selectivity. At the $k^{t h}$ event, SCM in Figure 2 computes the selectivity change of the preceding operator as follows:

$$
\Delta s(k)=s(k)-s(k-1)
$$

In this equation, $s(k)$ is the selectivity of the preceding operator computed at the $k^{t h}$ event. Thus, $0 \leq s(k) \leq 1$. Based on $\Delta s(k)$, SCM modifies $d^{\prime}(k)$, if necessary, to expedite the convergence of the queue length to $Q_{s}$ based on the selectivity information. For example, $\Delta s(k)<0$, if the selectivity of the operator pushing a data stream into the queue is decreasing. In this case, SCM adjusts the drop probability to shed fewer incoming stream tuples by making $d(k)<d^{\prime}(k)$ (or vice versa). In this way, it intends to avoid unnecessary data losses by dropping just enough tuples needed to closely support $Q_{s}$.

\section{Application of Fuzzy Control to Manage PER-STREAM BACKLOG}

Figure 3 shows the structure of our closed-loop load shedding scheme consisting of QCM and SCM. In fuzzy control, crisp variables expressed as real numbers, e.g., $e(k)$ and $f(k)$ in Figure 3, are mapped to linguistic variables, e.g., the error and trend, to naturally express human thoughts via the process called fuzzification. Using the linguistic variables, 


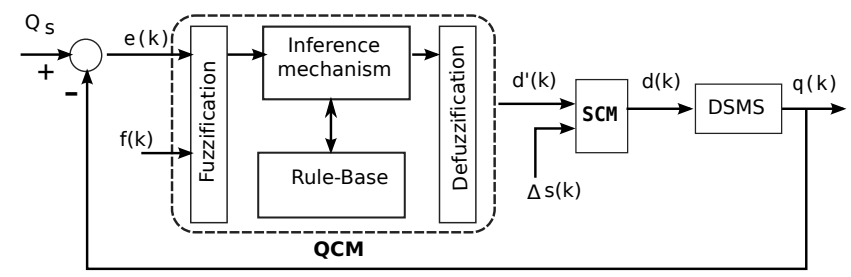

Fig. 3. Load Shedding via Fuzzy Logic Control

the inference scheme looks up the relevant if-precedent-thenconsequent rules. Finally, the defuzzification interface, such as the one in Figure 3, derives a crisp control signal from the linguistic rules. ${ }^{4}$

In the rest of this section, we describe the key observations that motivated the design of our fuzzy if-then rules for effective per-stream load shedding. Also, we describe the detailed procedure for fuzzy control, while giving illustrative examples.

\section{A. Design of Fuzzy Rules for Adaptive Load Shedding}

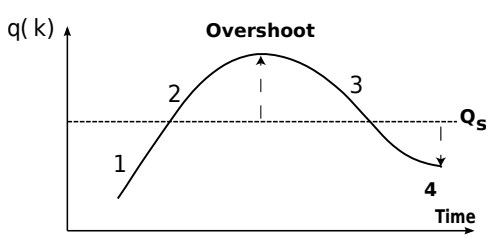

Fig. 4. Queue Length Variations

\begin{tabular}{|c|c|c|c|c|c|c|c|}
\hline \multirow{2}{*}{\multicolumn{2}{|c|}{ State 3}} & \multicolumn{6}{|c|}{$f(k)$} \\
\hline & & SD & MD & FD & FI & MI & SI \\
\hline \multirow{7}{*}{$e(k)$} & NL & $\overline{\mathrm{DM}}$ & $\overline{D M}$ & $\overline{D S}$ & IDM & $\mathrm{DM}$ & $\mathrm{DM}$ \\
\hline & NM & DS & $\mathrm{DS}$ & $\mathrm{DF}$ & DM & $\mathrm{DM}$ & DS \\
\hline & NS & \begin{tabular}{|l}
$\mathrm{DN}$ \\
\end{tabular} & $\overline{D F}$ & $\overline{D F}$ & DM & $\mathrm{DS}$ & DFI \\
\hline & $\mathrm{ZE}$ & $D N$ & $\mathrm{DN}$ & $\mathrm{DN}$ & IDS & $\overline{D S}$ & $\overrightarrow{D F}$ \\
\hline & PS & $\mathrm{DN}$ & $\mathrm{DN}$ & DNI & DF & $\mathrm{DF}$ & $\mathrm{DN}$ \\
\hline & PM & I DN & $\mathrm{DN}$ & $\mathrm{DN}_{\mathrm{H}}^{\prime}$ & DN & $\mathrm{DN}$ & $\mathrm{DN}$ \\
\hline & PL & \begin{tabular}{|l}
$\mathrm{DN}$ \\
\end{tabular} & $\mathrm{DN}$ & $\mathrm{DN}^{1}$ & DN & $\mathrm{DN}$ & $\mathrm{DN}$ \\
\hline
\end{tabular}

Fig. 5. Rule-base of QCM used to derive $d^{\prime}(k)$ from $e(k)$ and $f(k)$

\begin{tabular}{|c|c|c|c|c|c|}
\cline { 3 - 5 } \multicolumn{2}{c|}{} & \multicolumn{4}{c|}{$d^{\prime}(\boldsymbol{k})$} \\
\cline { 3 - 6 } \multicolumn{2}{c|}{} & DN & DF & DS & DM \\
\hline \multirow{3}{*}{$\Delta s(k)$} & PL & IDS & DS & DS & DM \\
\cline { 2 - 6 } & PM & IDS & DS & DS & DM \\
\cline { 2 - 6 } & PS & IDF & DS & DS & DM \\
\hline
\end{tabular}

Fig. 6. Rule-base of SCM used to derive $d(k)$ from $\Delta s(k)$ and $d^{\prime}(k)$

For effective fuzzy control, it is essential to design an effective rule-base. They are designed based on the logical understanding of the characteristics of the controlled system such as a DSMS. Figures 5 and 6 are the rule-bases used

\footnotetext{
${ }^{4}$ The fuzzification/defuzzification interface, inference scheme, and rule-base of SCM are not shown in Figure 3 to avoid a graphical repetition.
}

by QCM and SCM, respectively. The rules are designed to support $Q_{s}$ even when the system status and workloads change dynamically. For example, the rule $\{N S, F I\} \rightarrow D M$ in Figure 5 requires the load shedder to drop most (DM) tuples of the stream $\left(I_{i j}\right)$, if the current error, i.e., the linguistic version of $e(k)$, is negative small (NS), i.e., $e(k)>Q_{s}$ by a small amount. However, the trend, i.e., the linguistic version of $f(k)$, is fast increasing (FI), i.e., $f(k) \gg 0$.

We design the rules in Figure 5 based on our observations depicted in Figure 4. In the figure, there are four major states that characterize dynamic queue length changes. In normal conditions, it is desired that the system is in states 1 or 4 in Figure 4. Queue length overshoots are observed in states 2 and 3; that is, the queue length is longer than $Q_{s}$. Also, state 2 is worse than state 3 , since the queue length is diverging from $Q_{s}$ in state 2, whereas it is converging to $Q_{s}$ in state 3. The objective of QCM is to avoid overshoots by adapting the drop probability based on the error and trend, which are the linguistic (i.e., fuzzified) versions of Eq 2 and Eq 5. More specifically, we design the rule-base in Figure 5 for QCM considering the four states in Figure 4 as follows.

State 1. $e(k) \geq 0$ and $f(k)>0$ : In this state, the queue is not longer than $Q_{S}$, but it is increasing. Thus, the linguistic error is zero or positive, while the trend is increasing.

- Case 1: The error is zero. In this case, the queue length is equal to $Q_{s}$. If the trend indicates a fast increase (FI) or medium (MI) of the error, we drop some (DS) tuples as shown in in Figure 5. On the other hand, we drop few (DF) tuples, if the trend is a small increase (SI).

- Case 2: The error is positive. In this case, the queue is shorter than $Q_{s}$. As this is a desirable state, we either drop few (DF) tuples or drop none (DN). If the error is positive small (PS), i.e., the queue is shorter than $Q_{s}$ by a small amount, and the trend is FI or MI, we drop few (DF) tuples. Otherwise, the rules recommend the DSMS to drop none (DN).

State 2. $e(k)<0$ and $f(k)>0$ : In this zone, the queue is longer than $Q_{S}$ and further increasing. To cancel the overshoot and divergence from $Q_{s}$, QCM recommends the DSMS to drop the largest fraction of tuples in this state. It derives the tuple drop probability considering the magnitudes of the error and trend. For example, when the error is negative medium (NM), the queue is longer than $Q_{s}$ by a medium amount. In this case, the rules $\{N M, S I\} \rightarrow D S$ and $\{N M, M I\} \rightarrow D M$ in Figure 5 require the DSMS to drop some (DS) and drop most (DM) tuples for the slow increase and medium increase of the queue length, respectively.

State 3. $e(k) \leq 0$ and $f(k)<0$ : In this zone, the queue length is longer than or equal to $Q_{s}$, but it is decreasing. Control rules should be carefully designed by comparing the magnitudes of the error and trend, since it is possible for them to cancel each other. For example, if the error is negative small (NS) and the queue length shows a slowly decreasing (SD) trend, no tuple will be dropped as shown in Figure 5. In this region, a higher drop probability is derived for a larger absolute 
value of the error and a slower decrease of the queue length or vice versa.

State 4. $e(k)>0$ and $f(k)<0$ : In this state, the actual queue length is shorter than the set-point and it is further decreasing. Since this is a desirable state, the QCM requires the DSMS to drop none of the tuples.

SCM aims to further decrease the possibility of a queue length overshoot. To achieve the objective, SCM uses the ruletable in Figure 6 to derive $d(k)$ based on $\Delta s(k)$ and $d^{\prime}(k)$ computed by QCM. More specifically, SCM amplifies $d^{\prime}(k)$ only when the selectivity is increasing, i.e., $\Delta s(k)=s(k)-$ $s(k-1)>0$. In this case, SCM amplifies $d^{\prime}(k)$ based on the magnitude of $\Delta s(k)$ and $d^{\prime}(k)$. Thus, the rule-base in Figure 6 only considers positive selectivity changes.

For example, suppose that the selectivity change is positive large (PL), but QCM produces a drop none (DN) signal. In this case, the rule $\{P L, D N\} \rightarrow D S$ in Figure 6 modifies the DN signal produced by the QCM to the drop some (DS) signal, because the preceding operator's selectivity is increasing largely and the length of the stream queue succeeding the operator is likely to increase as a result.

Notably, the first two columns in Figure 6 require the system to further increase the drop probability considering the selectivity increase. On the other hand, the last two columns require no further increase of the drop probability, since the probability computed by QCM is already high enough in these cases. In this way, SCM aims to avoid overshoots and excessive data losses simultaneously.

\section{B. Fuzzy Control Procedure}

In this section, the fuzzification, inference, and defuzzification process for QCM is described. The procedure used by SCM is similar and omitted due to space limitations.

At the $k^{t h}$ control event, the fuzzification interface of QCM in Figure 3 maps $e(k)$ and $f(k)$ to the linguistic variables of the error and trend with the corresponding linguistic values. Using the derived linguistic values, the inference mechanism in in Figure 3 looks up the rule-base to find relevant if-then rules. From the relevant rules, the defuzzification interface of QCM derives a crisp control signal $d^{\prime}(k)$, via defuzzification, to support the desired per-stream backlog bound $Q_{s}$.

For fuzzy control, each variable, such as the variables in Figures 7(a) - 7(c), should be associated with the universe of discourse, i.e., the domain, [9]. In this paper, the universe of discourse for $e(k)$ and $\Delta s(k)$ is $[-0.5,0.5]$, while the universe of discourse for $f(k)$ is $[-3.5,3.5] .{ }^{5}$ For the control output, i.e., $d^{\prime}(k)$ and $d(k)$, we use the universe of discourse of [0, 0.95] to bound the range of the drop probability.

In fuzzy set theory underlying fuzzy control theory, to deal with uncertainties, a crisp value is mapped to one or more fuzzy sets; that is, partial membership is allowed unlike set theory that only supports binary membership [9]. The degree of membership, called certainty, is computed using

\footnotetext{
${ }^{5}$ If $e(k)$ or $\Delta s(k)$ is smaller than -0.5 (larger than 0.5), it belongs to NL (PL) with certainty 1 . Similarly, if $f(k)<-3.5(f(k)>3.5)$, it belongs to SD (SI) with certainty 1.
}

membership functions (MFs). In this paper, $e(k)$ may belong to one or two fuzzy MFs in Figure 7(a), because we design adjacent MFs to overlap. For all the MFs in Figure 7(a), except for the leftmost and rightmost ones, we use symmetric triangles of an equal base and 50\% overlap with the adjacent fuzzy MFs. In Figure 7(a), the horizontal axis represents $e(k)$, while the vertical axis indicates the certainty of the membership.

Fuzzification is the first step in fuzzy control. It maps the input crisp variables to the corresponding linguistic variables. In this paper, $e(k)$ measured at the $k^{\text {th }}$ control event are fuzzified to the linguistic error with one or two linguistic values in Figure 7(a): Negative Large, Negative Medium, Negative Small, ZEro, Positive Small, Positive Medium, and Positive Large. If $e(k)<0$, it is mapped to one or more Negative MFs in Figure 7(a) or vice versa.

Via fuzzification, we also map $f(k)$ to the linguistic trend with one or two of the linguistic values in Figure 7(b): Slow Decrease, Medium Decrease, Fast Decrease, Slow Increase, Medium Increase, and Fast Increase. If $f(k)<0$, it is mapped to one or two Decrease MFs in Figure 7(b) or vice versa (Eq 5). In the following example, QCM fuzzifies $e(k)$ and $f(k)$.

Example 1. Fuzzification. Let $e(k)=-0.15625$ and $f(k)=1.5$. In this case, the error, i.e., the linguistic version of $e(k)$, belongs to NM and NS in Figure 7(a) with certainty $\mu_{N M}(-0.15625)=0.25$ and $\mu_{N S}(-0.15625)=0.75$. At the same time, the trend, i.e., the linguistic version of $f(k)$, belongs to MI in Figure 7(b) with certainty 1.

Using the fuzzified linguistic error and trend, the inference mechanism of QCM looks up the rule-base in Figure 5 to find the related if-then rules. The linguistic variables are used to infer the if-then rules. Due to partial membership allowed in fuzzy control, more than one if-then rules can be triggered as a result of the rule-base look-up. In this case, the certainty of an inferred rule is set to the minimum of the certainty values of the precedents, because the consequent cannot be more certain than the precedents [9].

Example 2. Rule-Base Lookup. In Example 1, the error belongs to $N S$ and $N M$ with the certainty 0.75 and 1 . Further, the trend belongs to $M I$ with the certainty 1 . In this case, two rules in Figure 5, i.e., $\{N S, M I\} \rightarrow D S$ and $\{N M, M I\} \rightarrow$ $D M$, are relevant. Since the error and trend belong to NS and MI with the certainty 0.75 and 1 , the certainty of the first rule $\{N S, M I\} \rightarrow D S$ is: $\min \{0.75,1\}=0.75$. At the same time, the error and trend belong to NM and MI with the certainty 0.25 and 1 ; therefore, the certainty of the second rule $\{N M, M I\} \rightarrow D M$ is: $\min \{0.25,1\}=0.25$.

Finally, the defuzzification interface derives crisp $d^{\prime}(k)$ from the rules. For defuzzification, QCM and SCM use the MFs in Figure 7(c): Drop None, Drop Few, Drop Some, and Drop Most. To describe the defuzzification process, let $x$ and $y$ represent the row and column indexes in Figure $5(1 \leq x \leq 7$ and $1 \leq y \leq 6)$. Also, let $\mu(x, y)$ denote the certainty of the corresponding rule $(x, y)$ in the table. Further, let $c(x, y)$ denote the center of the fuzzy set that is the rule $(x, y)$ 's 


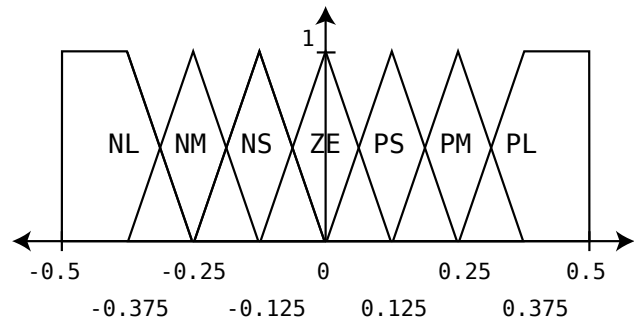

(a) MFs used to fuzzify $e(k)$ or $\Delta s(k)$

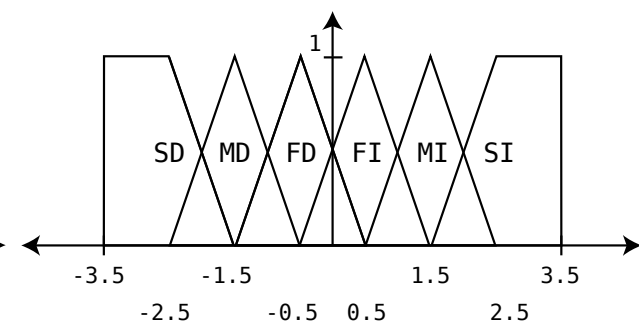

(b) MFs used to fuzzify $f(k)$

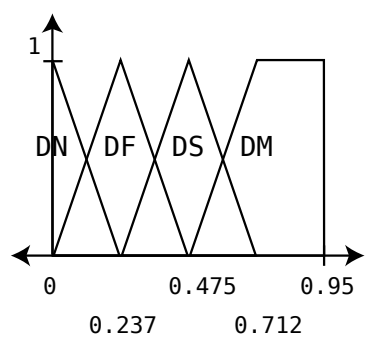

(c) MFs used to derive $d^{\prime}(k)$ and $d(k)$

Fig. 7. Membership Functions (MFs) for Fuzzification and Defuzzification

consequent. For a triangular fuzzy set, the center is the middle of the triangle's base. In this paper, we use the centroid method [9] to compute the drop probability for per-stream queue length control:

$$
d^{\prime}(k)=\frac{\sum_{x, y} \mu(x, y) \cdot c(x, y)}{\sum_{x, y} \mu(x, y)}
$$

Example 3. Defuzzification. In Example 2, two control signals $D S$ and $D M$ are derived with certainty values 0.75 and 0.25 , respectively. According to Figure 7(c), the center of $D S$ is 0.475 and the center of $D M$ is 0.712 . Using the certainty values of the rules from the previous example, we compute the drop probability: $d^{\prime}(k)=\frac{0.75 \cdot 0.475+0.25 \cdot 0.712}{0.75+0.25}=0.534$. Thus, this stream's tuples will be randomly dropped with probability 0.534 until the next control event happens, unless SCM further adjusts the drop probability based on the selectivity change $\Delta s(k)$.

\section{Performance Evaluation}

In this section, a description of the experimental settings is followed by the performance evaluation results.

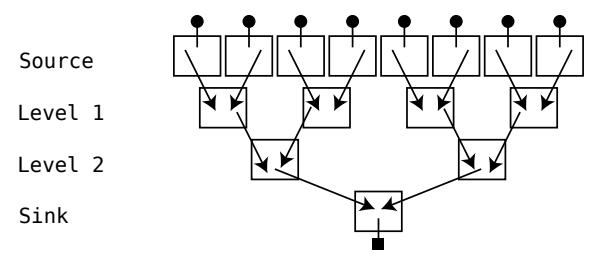

Fig. 8. Query network topology for experiments

For our experiments, we use CAIDA's Internet traffic traces collected by the equinix-sanjose high-speed monitor [17]. The average bit-rate of the monitored network is around 2.3 Gbps. Approximately 500,000 packets on average are being processed every second by each of the micro-benchmark applications that we have used for performance evaluation. Thus, our applications model the challenging problem of monitoring bursty and highly dynamic Internet traffic patterns.

Figure 8 shows the query network topology used in our experiments. The input data streams flow from the sources and processed in the four layers to generate the query results at the sink. The 15 physical machines in the figure are connected via a $1 \mathrm{Gbps}$ switch. Each machine has a $1.5 \mathrm{GHz}$ or $2.2 \mathrm{GHz}$ Intel dual core CPU and 1GB main memory. In our experiments, the stream sources use the faster machines to generate highrate data streams. Input data are stored in raw packet format and partitioned to eight source level machines based on their destination addresses, modeling network monitoring sensors placed at different locations. Sources read blocks of the traces, transform the data into Borealis tuples, and inject them into the input streams of the query network. The 8 query chains in Figure 8 are executed in parallel. For each query operator, we use a jumping window of 1000 tuples. Thus, an individual stream query operator is executed for every 1000 tuple arrivals.

Table I shows the flow statistics (FS) application. In FS, the flow information, including source and destination addresses as well as port numbers, is extracted from the TCP/IP packets to continuously analyze flow statistics for the sourcedestination pairs found in the jumping window. (For brevity, the window constraint is not included in the table.) The continuous queries in the table are deployed at the specified levels in Figure 8 to analyze network traffic patterns based on flow IDs. The sink node computes a running average and reports flows whose rates are higher than the value specified in Table I. Although we have done experiments for other network analysis applications, we mainly report the results of FS due to space limitations.

In this paper, we compare the performance of our approach, called FuzzyQS, to that of Borealis, which employs FIT [16] for load shedding, mainly in terms of the queue length and CQ processing delay. At every second, we record the maximum queue length measured in the query network. In this paper, we set $Q_{S}=1000$ tuples for performance evaluation purposes. For performance evaluation, we keep track of the biggest queue size in the whole distributed DSMS at every second. In addition, the largest source-to-sink query processing delay is measured every 1s. Since our approach avoids overloads via load shedding on a per-stream and per-operator basis, they can indirectly reduce the delay too.

Figure 9 shows the cumulative distribution of the queue length for 10 experimental runs of the FS application. As shown in the figure, the 95 percentile queue length of Borealis and FuzzyQS is 59435 and 857 tuples, respectively. Figure 10 shows the transient queue length measured every 1s for 1 run. FuzzyQS controls the per-stream queue length to be below $Q_{S}=1000$ tuples for most of the time except for a few 


\begin{tabular}{|c|c|c|}
\hline DSMS Application & Level & Query \\
\hline Flow Summary (FS) & Source & $\begin{array}{l}\text { select * from (select count (*), sum(size), flowid from (select [flow fields] } \\
\text { from input) group by flowid) where count > } 5 \\
\text { select * from (select sum(count), sum(size), flowid from (select * from } \\
\text { input } 1 \text {, input } 2 \text { ) group by flowid) where count > } 10\end{array}$ \\
\hline & $\begin{array}{l}\text { Level } 2 \\
\text { Sink }\end{array}$ & $\begin{array}{l}\text { select * from (select sum(count), sum(size), flowid from (select * from } \\
\text { input1, input2) group by flowid) where count > } 20 \\
\text { select * from (select sum(count), sum(size), flowid from (select * from } \\
\text { input1, input2) group by flowid) where size > avg(size) / } 2\end{array}$ \\
\hline
\end{tabular}

TABLE I

FLOW ANALYSIS APPLICATION DEPLOYED OVER THE QUERY NETWORK IN FIGURE 8

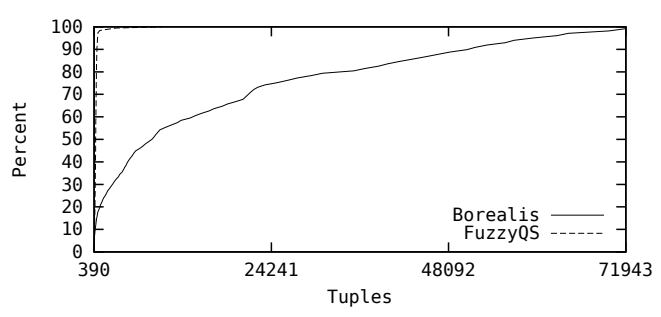

Fig. 9. Flow Summary: Cumulative Queue Length for 10 Runs

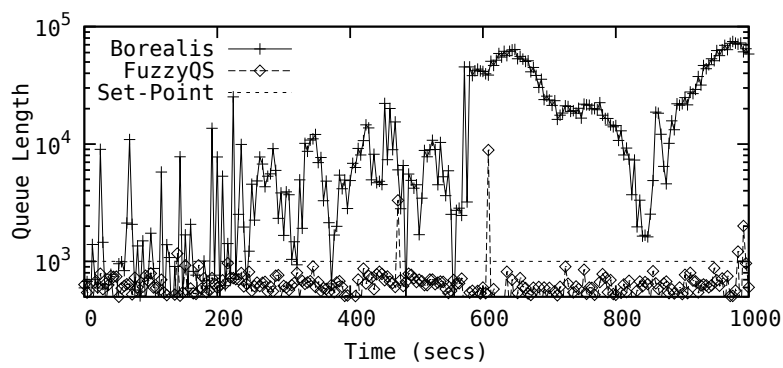

Fig. 10. Flow Summary: Transient Queue Length for 1 Run

overshoots as shown in Figure 10. From Figures 9 and 10, we observe that the per-stream queue length of FuzzyQS is up to two orders of magnitude shorter than that of Borealis. By controlling the per-stream backlog, FuzzyQS considerably decreases the delay too. In Figure 11, the 95 percentile delay of Borealis and FuzzyQS for 10 runs is $8.86 \mathrm{~s}$ and 5.26s, respectively. Thus, the 95 percentile delay of our approach is approximately $40 \%$ shorter than that of Borealis. FuzzyQS achieves these improvements via per-stream, fully-distributed queue length control based on fuzzy rules. Borealis makes system-wide decisions on load shedding at the nodes near the sinks. The decisions are aggregated and cascaded back toward the sources in an incremental manner [16]. Notably, existing DSMS, including [1], [2], [3], [5], [4], [6], [18], [19], [13], [14], [15], [8], [16], do not aim to support the desired per-stream queue length even in the presence of dynamic workloads. In the future, we will investigate how to further reduce per-stream queue length overshoots.

Figure 12 shows the cumulative output rate for 10 runs, which measures the number of the output tuples leaving the sink per second. From the figure, we observe that the output

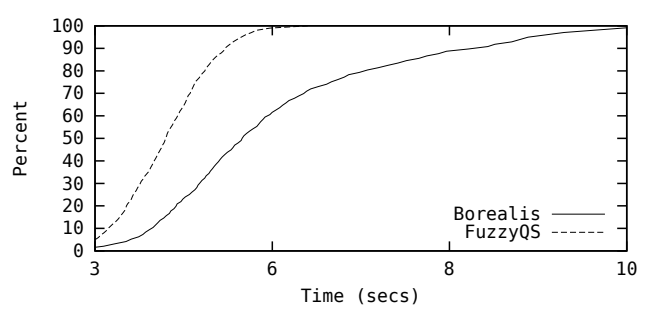

Fig. 11. Flow Summary: Cumulative Delay for 10 Runs

rate of FuzzyQS is similar to the rate of Borealis. Thus, our approach does not simply drop more tuples than Borealis does to control the queue length. Instead, it judiciously adapts the drop rate of each stream, if necessary, to avoid forming a bottleneck in the query chains.

In addition to FS, we have designed and run two more applications to analyze network traffic patterns based on the source and destination IP address, respectively. In these experiments, FuzzyQS has closely supported $Q_{s}$. The queue length of Borealis is up to an order of magnitude longer that that of FuzzyQS. The response time of Borealis is longer than ours by $0.63 \mathrm{~s}-1.2 \mathrm{~s}$. Also, FuzzyQS has shown simliar output rates to those provided by Borealis. Detailed results for these DSMS applications are not included due to space limitations.

Moreover, our approach incurs little overhead. In our experiments, our load shedding scheme is activated approximately 80 times/s on average, consuming approximately $0.25 \%$ total CPU utilization. It consumes less than $1 \mathrm{~KB}$ of memory in each node to store the two rule-bases and keep track of the queue lengths and operator selectivities.

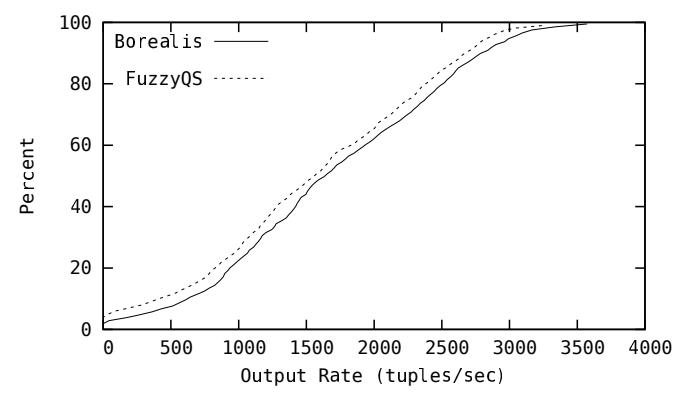

Fig. 12. Flow Summary: Cumulative Output Rates for 10 Runs 


\section{RELATED WORK}

Load shedding techniques have been studied to enhance the performance and reliability of DSMS [13], [14], [8], [16]. Under overload, the load shedder of Aurora drops the tuple with the lowest utility first under the assumption that the utilities of streams are known a priori [8]. In Borealis, global load shedding decisions are made near the sink and propagated back toward the stream sources [16]. Data Triage does load shedding under overload, while generating short summaries of the discarded packets [13]. This approach is complementary to ours in that it could be combined with our approach to support a summary of dropped tuples in the background. Overall, our work is different in that it supports fully localized, per-stream backlog management by leveraging continuous query semantics, while applying well-established fuzzy control techniques.

A proportional and integral (PI) controller is designed to bound the average per-tuple delay via load shedding [14]. It has also been used to support real-time periodic queries [18], [19], [23] implemented in STREAM [5]. However, a PI controller may largely fail to support the desired performance, if the model of the controlled system, e.g., a DSMS, derived offline using representative workloads becomes inaccurate due to dynamic workloads and system behaviors [10]. Unfortunately, finding representative workloads for a highlydynamic, nonlinear systems, such as DSMS, is very hard (if at all possible). Applying other branches of control theoretic techniques based on mathematical modeling of the controlled system, such as [11], [12], also suffers from the difficulty of accurately modeling highly-dynamic and nonlinear DSMS.

Fuzzy logic control has been applied to manage the performance of other computational systems such as real-time tracking [24]. Basaran et al. [25] show that fuzzy control considerably outperforms a PI controller and a model predictive controller in terms of CPU utilization control in a real-time operating system. However, these approaches do not aim to support load shedding considering distributed DSMS semantics discussed in Section I. Nor do they support eventdriven control to manage the per-stream backlog.

\section{CONCLUSION AND Future WORK}

In this paper, we present a lightweight load shedding scheme to manage the per-stream backlog. Our approach directly considers the per-stream backlog and selectivity of each query operator to support graceful, fine-grained load shedding in a distributed manner. Further, our approach is event-driven. It quickly reacts to bursty workloads, while avoiding an unnecessary activation of the controller under light load conditions. The performance of our approach is evaluated thoroughly using high-rate Internet traces by extending Borealis, which is an advanced open-source distributed DSMS. From our experiments, we observe that our approach can closely support the desired per-stream queue length, while considerably decreasing the per-stream backlog and query processing delay compared to Borealis. In the future, we will explore more effective scheduling and load shedding schemes to further enhance the performance of distributed DSMS.

\section{ACKNOWLEDGEMENT}

This work was supported, in part, by the NSF grant CNS117352 and supplementary project to AFOSR grant FA955009-1-0165.

\section{REFERENCES}

[1] "StreamBase." [Online]. Available: http://www.streambase.com/

[2] "InfoSphere Streams." [Online]. Available: http://www-01.ibm.com/ software/data/infosphere/streams/

[3] "Microsoft StreamInsight." [Online]. Available: http://msdn.microsoft. com/en-us/library/ee362541.aspx

[4] D. Abadi, Y. Ahmad, M. Balazinska, U. Çetintemel, M. Cherniack, J. Hwang, W. Lindner, A. Maskey, A. Rasin, E. Ryvkina, N. Tatbul, Y. Xing, and S. Zdonik, "The Design of the Borealis Stream Processing Engine," in CIDR, 2005.

[5] R. Motwani, J. Widom, A. Arasu, B. Babcock, S. Babu, M. Datar, G. S. Manku, C. Olston, J. Rosenstein, and R. Varma, "Query Processing, Approximation, and Resource Management in a Data Stream Management System," in CIDR, 2003.

[6] S. Chandrasekaran, O. Cooper, A. Deshpande, M. J. Franklin, J. M. Hellerstein, W. Hong, S. Krishnamurthy, S. R. Madden, F. Reiss, and M. A. Shah, "TelegraphCQ: Continuous Dataflow Processing," in SIGMOD, 2003

[7] N. Tatbul, "Load shedding techniques for data stream management systems," Ph.D. dissertation, Brown University, 2007.

[8] N. Tatbul, U. Çetintemel, S. Zdonik, M. Cherniack, and M. Stonebraker, "Load Shedding in a Data Stream Manager," in $V L D B, 2003$.

[9] W. Pedrycz, Fuzzy Control and Fuzzy Systems. Wiley and Sons, Inc., 1993.

[10] C. L. Phillips and H. T. Nagle, Digital Control System Analysis and Design. Prentice Hall, 1995.

[11] K. J. A. ström and B. Wittenmark, Adaptive Control. Addision Wesley, 1995.

[12] J. Maciejowski, Predictive Control with Constraints. Prentice Hall 2002.

[13] F. Reiss, "Declarative Network Monitoring with an Underprovisioned Query Processor," in ICDE, 2006.

[14] Y.-C. Tu, S. Liu, S. Prabhakar, and B. Yao, "Load Shedding in Stream Databases: A Control-Based Approach,” in VLDB, 2006, pp. 787-798.

[15] B. Mozafari and C. Zaniolo, "Optimal Load Shedding with Aggregates and Mining Queries," in ICDE, 2010.

[16] N. Tatbul, U. Çetintemel, and S. B. Zdonik, "Staying FIT: Efficient Load Shedding Techniques for Distributed Stream Processing," in VLDB, 2007.

[17] K. C. Claffy, D. Andersen, and P. Hick. The CAIDA Anonymized 2012 Internet Traces. [Online]. Available: http://www.caida.org/data/passive/ passive_2012_dataset.xml

[18] Y. Wei, S. H. Son, and J. A. Stankovic, "RTSTREAM: Real-Time Query Processing for Data Streams," in ISORC, 2006.

[19] K. Kapitanova, Y. Wei, W. Kang, and S. H. Son, "Applying Formal Methods to Modeling and Analysis of Real-time Data Streams," Journal of Computing Science and Engineering, vol. 5, no. 1, 2011.

[20] "Linear Road: A Stream Data Management Benchmark," http://pages.cs.brandeis.edu/ linearroad/.

[21] "1998 World Cup Web Site Access Logs," http://ita.ee.lbl.gov/html/contrib/WorldCup.html.

[22] F. Reiss, "Data Triage: An Adaptive Architecture for Load Shedding in TelegraphCQ," in ICDE, 2005.

[23] Y. Zhang, C. Huang, and D. Zhang, "A Novel Adaptive Load Shedding Scheme for Data Stream Processing," in Future Generation Communication and Networking, vol. 1, 2007.

[24] B. Li and K. Nahrstedt, "A Control-Based Middleware Framework for Quality of Service Adaptations," Journal on Selected Areas in Communications, vol. 17, no. 9, 1999.

[25] C. Basaran, M. H. Suzer, K. D. Kang, and X. Liu, "Robust Fuzzy CPU Utilization Control for Dynamic Workloads," Journal of Systems and Software, vol. 83, no. 7, pp. 1192-1204, 2010. 